\title{
A STUDY OF DYSLIPIDAEMIA IN LICHEN PLANUS PATIENTS
}

\author{
Sudhesan Athira ${ }^{1}$, Anitha Krishna ${ }^{2}$
}

${ }^{1}$ Lecturer, Department of Dermatology \& Venereology, Government T. D. Medical College, Alappuzha.

${ }^{2}$ Associate Professor, Department of Dermatology \& Venereology, Government T. D. Medical College, Alappuzha.

\section{ABSTRACT}

\section{BACKGROUND}

Lichen planus (LP) is a distinct autoimmune inflammatory, papulosquamous skin disorder. Some studies have showed an increased prevalence of dyslipidaemia in lichen planus patients. Disturbances in the lipid metabolism due to underlying inflammation and oxidative stress which releases inflammatory cytokines could be accountable for this.

Aim of the study was to find out the proportion of dyslipidaemia among lichen planus patients and compare the clinical pattern of lichen planus in patients having dyslipidaemia.

\section{MATERIALS AND METHODS}

This cross-sectional descriptive study was done on 75 lichen planus patients who attended a tertiary care hospital in Kerala. The diagnosis of LP was made by clinical examination and confirmed histopathologically in doubtful cases. Fasting serum was analysed for cholesterol, triglycerides (TG), high density lipoprotein (HDL) and low-density lipoprotein (LDL). The presence of dyslipidaemia was established, based on the National Cholesterol Education Programme Adult Treatment Panel III definitions (NCEP-ATP III criteria).

\section{RESULTS}

The study showed that the proportion of dyslipidaemia in lichen planus was $41.3 \%$ and patients with extensive skin lesions showed higher prevalence of dyslipidaemia.

\section{CONCLUSION}

This study demonstrated that a significant proportion of lichen planus patients showed high lipid levels in blood and for patients presenting with LP, physicians should consider screening them for dyslipidaemia.

\section{KEYWORDS}

Lichen Planus, Dyslipidaemia, Chronic Inflammation, Oxidative Stress.

HOW TO CITE THIS ARTICLE: Athira S, Krishna A. A study of dyslipidaemia in lichen planus patients. J. Evolution Med. Dent. Sci. 2017;6(42):3288-3291, DOI: 10.14260/Jemds/2017/712

\section{BACKGROUND}

Lichen planus (LP) is a unique idiopathic chronic inflammatory papulosquamous disorder that affects the skin, mucous membrane, nails and hair. L P initially presents as small, violaceous, flat-topped, polygonal papules mostly on the extremities. The overall prevalence of LP is estimated to be between $0.14 \%$ and $0.80 \%$ worldwide. Cell mediated immunity plays a major role in the pathogenesis of lichen planus. Activated T- cells release cytokines leading to attraction of inflammatory cells and apoptosis of keratinocytes. ${ }^{1}$

Dyslipidaemia means an abnormal amount of lipids in the blood. Dyslipidaemia has been found to be associated with some chronic inflammatory skin diseases like psoriasis. Recently, various studies have shown an increased incidence of dyslipidaemia among lichen planus patients. Chronic inflammation and defective antioxidant defence mechanisms

Financial or Other, Competing Interest: None.

Submission 20-04-2017, Peer Review 13-05-2017,

Acceptance 20-05-2017, Published 25-05-2017.

Corresponding Author:

Dr. Sudhesan Athira,

Lecturer,

Department of Dermatology \& Venereology,

Government T. D. Medical College,

Vandanam,

Alappuzha-688005, Kerala.

E-mail: athirasudhesan@gmail.com

DOI: $10.14260 /$ jemds $/ 2017 / 712$ have been implicated in the pathogenesis of altered lipid levels in lichen planus. Cytokines (TNF-alpha, Interleukin-2, Interleukin-6) which are released as a part of chronic inflammatory process have been implicated in the lipid profile abnormalities in these patients. ${ }^{2}$

\section{MATERIALS AND METHODS}

This cross-sectional descriptive study was done on 75 lichen planus patients who attended a tertiary care hospital in Kerala, after getting informed written consent. Aim was to find out the proportion of dyslipidaemia among lichen planus patients and to compare the clinical pattern of lichen planus in patients having dyslipidaemia. Patients of all age groups with clinical diagnosis of lichen planus, confirmed by skin biopsy in doubtful cases were enrolled in the study. Patients with a documented history of diabetes mellitus, systemic hypertension, liver diseases, renal diseases, thyroid diseases, other chronic inflammatory diseases like rheumatoid arthritis, systemic lupus erythematosus, obese patients, patients receiving treatment with systemic corticosteroids, retinoic acid, methotrexate, chloroquine and hormone replacement therapy were excluded from the study.

Routine blood and urine examination, fasting and postprandial blood sugar, renal function test, liver function test, thyroid function test (TFT), and antinuclear antibody (ANA), were done in all patients. Fasting lipid profile levels were done and recorded in the proforma. Total cholesterol and triglycerides were estimated by enzymatic method and 
HDL estimated by precipitation method. LDL was calculated by the formula, total cholesterol-(TG/5+HDL).

The diagnosis of dyslipidaemia was established by the presence of one of the following parameters: triglycerides $>150 \mathrm{mg} / \mathrm{dL}$; total cholesterol $>200 \mathrm{mg} / \mathrm{dL}$; LDL >130 $\mathrm{mg} / \mathrm{dL} ; \mathrm{HDL}<40 \mathrm{mg} / \mathrm{dL}$ for males and $<50 \mathrm{mg} / \mathrm{dL}$ for females after 9 to 12-hour fast, based on the National Cholesterol Education Programme Adult Treatment Panel III definitions (NCEP-ATP III criteria).

Data was analysed in terms of frequency, proportions and percentage by using appropriate biostatistics. Various parameters like gender, age, duration of disease, distribution of lesions, morphology of lesions, mucosal involvement, nail involvement in patients with dyslipidaemia were compared with those without dyslipidaemia using appropriate statistical test using SPSS software ( $\chi^{2}$ - test and unpaired Ttest). Effect modification was identified when the P-value was $<0.05$.

\section{RESULTS}

Out of the 75 lichen planus patients, 31 patients (41.3\%) had dyslipidaemia.

\section{No dyslipidemia $\square$ Dyslipidemia present}

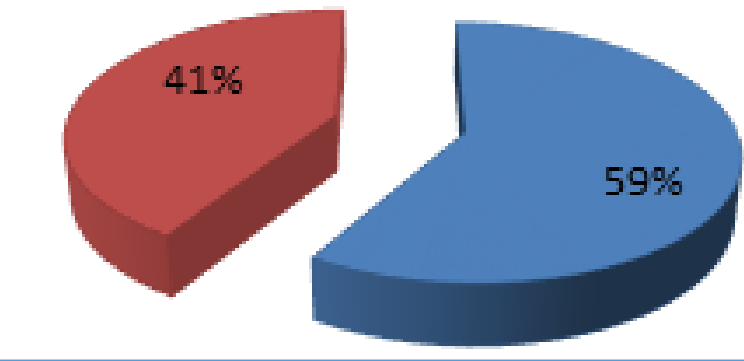

Figure 1. Dyslipidaemia in Lichen Planus Patients

Out of the total lichen planus with dyslipidaemia, 34.3\% were male and $47.5 \%$ were female. There was no statistically significant difference in the prevalence of dyslipidaemia across gender. (Chi -square test, $P$ value 0.246 ).

\begin{tabular}{|c|c|c|}
\hline \multirow{2}{*}{ Gender } & \multicolumn{2}{|c|}{ Dyslipidaemia } \\
\cline { 2 - 3 } & Present & Absent \\
\hline Male & $34.3 \%$ & $65.7 \%$ \\
\hline Female & $47.5 \%$ & $52.5 \%$ \\
\hline \multicolumn{2}{|c|}{ Table 1. Dyslipidaemia across Gender in Lichen Planus } \\
Patients \\
\hline
\end{tabular}

26 of 31 patients with dyslipidaemia (83.9\%) were in the age group of $>40 \mathrm{yrs}$. It is statistically significant with $P$ value 0.005 (Pearson Chi-Square test).

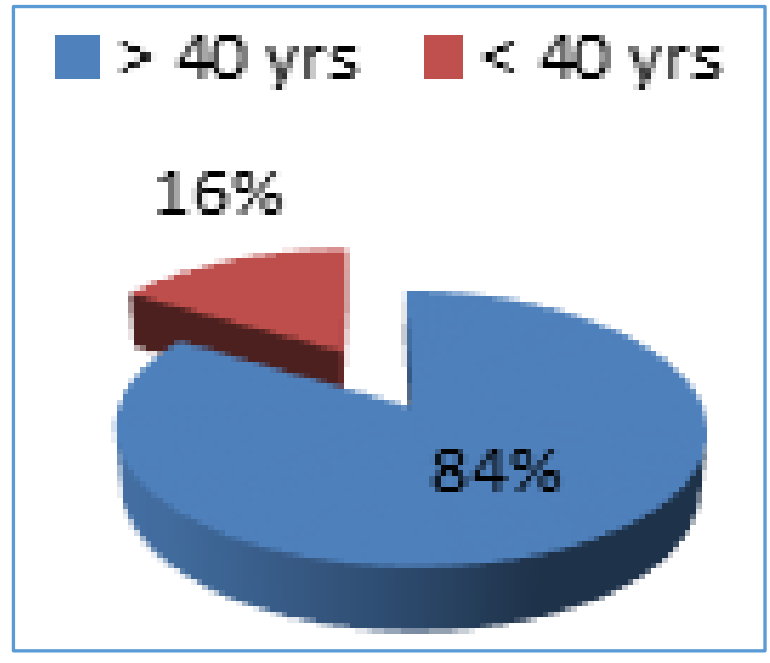

Figure 2. Age Distribution in Lichen Planus Patients with Dyslipidaemia

Mean age of the patients having dyslipidaemia was 52.65 with a standard deviation of 14.584 and the mean age of those without dyslipidaemia was 37.45 with a standard deviation of 15.093. There was a statistically significant association between the age and dyslipidaemia in lichen planus patients (unpaired T-test, $p$ value $<0.001$ ).

Duration of disease in dyslipidaemia patients ranged from $<6$ months to 8 years. There was no statistically significant association between duration of Lichen planus and dyslipidaemia (P value 0.618, Pearson Chi-Square test).

\begin{tabular}{|c|c|c|}
\hline \multirow{2}{*}{$\begin{array}{c}\text { Duration of } \\
\text { Lichen Planus }\end{array}$} & \multicolumn{2}{|c|}{ Dyslipidaemia } \\
\cline { 2 - 3 } & Present & Absent \\
\hline 0-6 months & $48.4 \%$ & $45.5 \%$ \\
\hline 6-12 months & $29 \%$ & $22.7 \%$ \\
\hline 1-3 years & $12.9 \%$ & $20.5 \%$ \\
\hline 3-5 years & $3.2 \%$ & $9.1 \%$ \\
\hline 5-8 years & $6.5 \%$ & $2.3 \%$ \\
\hline \multicolumn{2}{|c|}{ Table 2. Duration of Lichen Planus and Dyslipidaemia } \\
\hline
\end{tabular}

45.2\% patients had lesions over the upper limb, lower limb and trunk. 25.8\% had involvement of upper limb and lower limb and $16.1 \%$ presented with lesions in the lower limb alone. There was no statistically significant correlation with dyslipidaemia and site of involvement ( $p$ value -0.638 ). But patients with extensive skin lesions showed more prevalence of dyslipidaemia.

Most common morphology of lichen planus in patients with dyslipidaemia was classical LP (71\%). There was 1 patient each in the categories of vesiculobullous and follicular LP. Both these patients had dyslipidaemia. There was no statistically significant association between the occurrence of dyslipidaemia and various morphological patterns of lichen planus (Chi-square test, P value 0.396).

We could not find any statistically significant association between mucosal lesions and dyslipidaemia ( $p$ value- 0.914 ).

$70 \%$ patients with nail involvement had dyslipidaemia. A significant correlation was noted with dyslipidaemia ( $p$ 0.048 ) and nail involvement. 


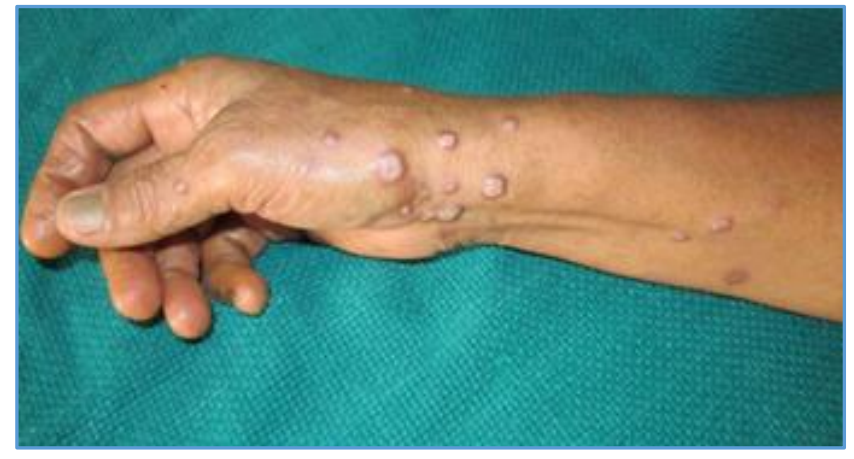

Picture 1. Classical Lichen Planus

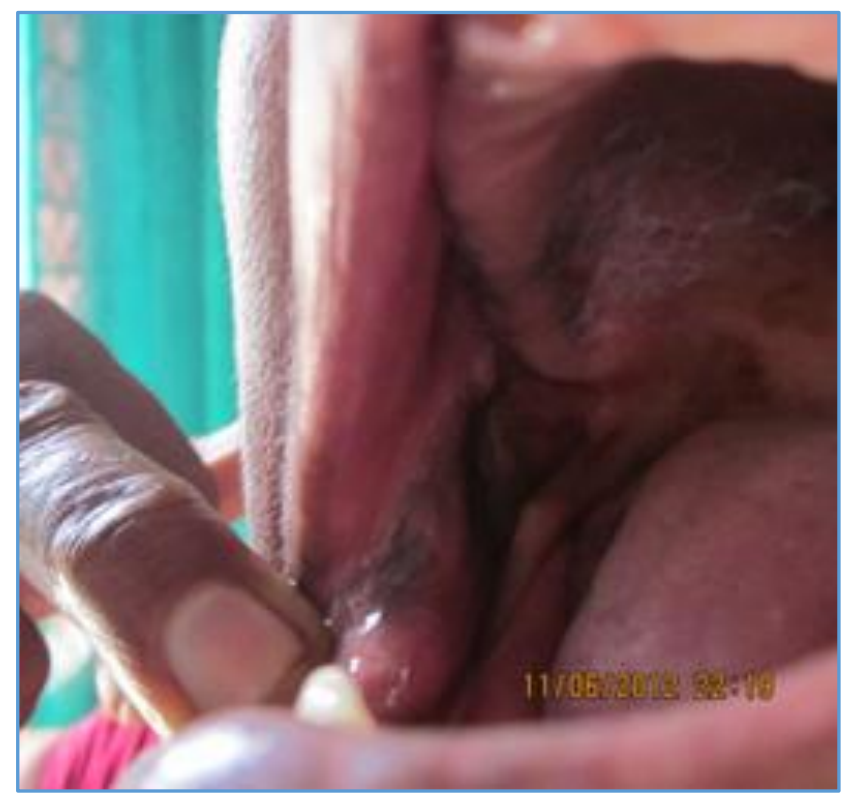

Picture 2. Oral Lichen Planus

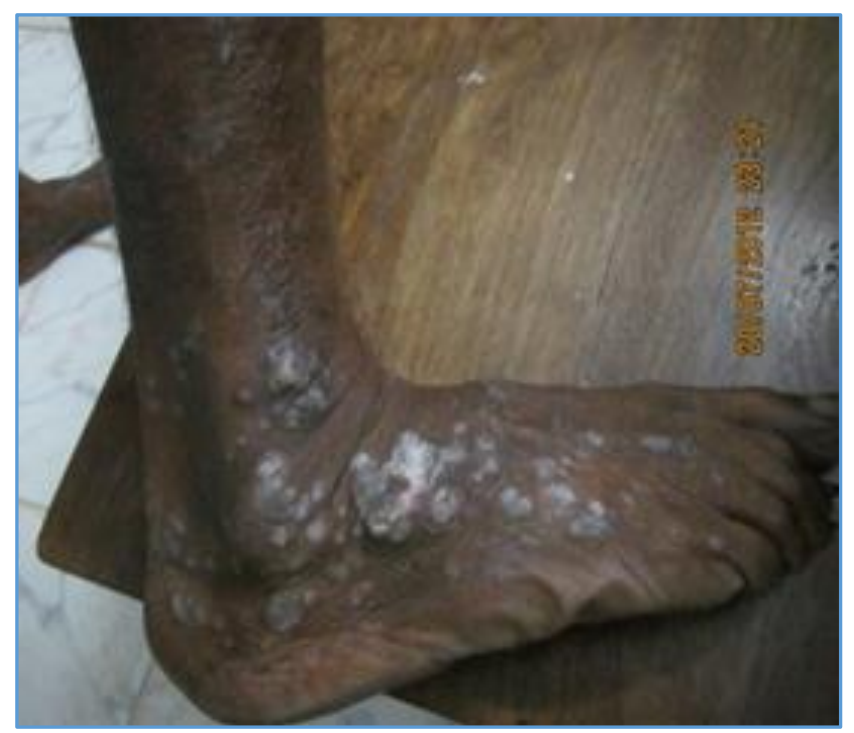

Picture 3. Hypertrophic Lichen Planus

\section{DISCUSSION}

In this study, dyslipidaemia was observed in $41.3 \%$ patients. Various studies have described a high prevalence of dyslipidaemia in patients with LP.2,3,4,5 Dreiher et al analysed the prevalence of dyslipidaemia among Israeli patients with LP and concluded that the prevalence of dyslipidaemia was significantly high in lichen planus patients (42.5\%) even after controlling confounders. ${ }^{3}$ In another case-control study by Arias-Santiago et al, the prevalence of dyslipidaemia in patients with lichen planus was $61 \%$, compared to $33 \%$ for controls. ${ }^{2}$ Chronic inflammation with a persistent elevation in the level of proinflammatory cytokines such as leptin, adiponectin, tumor necrosis factor- $\alpha$ (TNF), interleukin- 6 (IL-6), monocyte chemotactic protein-1 (MCP-1), and other adipocytokines produced by adipocytes are shown to be associated with metabolic complications such as dyslipidaemia and are elevated in many dermatological diseases associated with chronic inflammation. ${ }^{6}$ Oxidative stress, a condition of relative imbalance between reactive oxygen species (ROS) and antioxidants, is believed to play a central role in the pathogenesis of metabolic dyslipidaemia.7,8 A study conducted by Aly et al in Egyptian patients with lichen planus showed that the serum levels of nitric oxide (NO) was higher in these patients, suggesting that oxidative stress resulting in the generation of ROS may play a role in the pathogenesis of LP. ${ }^{9}$ One of the major presentations of oxidative stress is peroxidation of lipid rich membranes. ${ }^{10}$ Malondialdehyde (MDA), the end product of lipid peroxidation, is elevated in LP patients. ${ }^{9}$

The inflammatory cellular infiltrate in LP is a well-known source of reactive oxygen species (ROS). ${ }^{11}$ A delayed hypersensitivity immune reaction, in which the release of cytokines by activated $T$ cells attracts inflammatory cells and leads to the destruction of keratinocytes, resulting in generation of reactive oxygen species, may play a role in the pathogenesis of LP.11,12,13,14 LP is characterised by the upregulation of inflammatory CXCR3 ligands associated with the recruitment of effector cytotoxic $T$ cells and plasmacytoid dendritic cells. ${ }^{12}$ Various cytokines including IL-2, IL-4, IL-6, IL-10, TNF- $\alpha$, interferon (IFN) $-\alpha$, IFN- $\gamma$ and transforming growth factor- $\beta 1$ are involved in LP.12,14 Induction of the IFN$\alpha$-inducible CXCR3 ligands, CXCL9, CXCL10 and CXCL11 has been reported. These inflammatory processes could potentially explain the link between LP and dyslipidaemia.

Our study showed that the prevalence of dyslipidaemia was same across the gender. Arias-Santiago et al reported a significantly high prevalence of dyslipidaemia in both men and women with lichen planus but there was no significant relation with gender ${ }^{2}$; whereas according to Dreiher et al the association was more among females. ${ }^{3}$

The present study showed that most of the patients with dyslipidaemia were in the older age group. Here, age must have acted as a confounding factor, because of higher prevalence of dyslipidaemia in older patients in general population.

In the present study, we could not find any significant association between duration of lichen planus and dyslipidaemia. Though there was no statistically significant association between the extent of skin lesions and dyslipidaemia, patients with extensive skin lesions showed a higher prevalence of dyslipidaemia.

There was no significant association between the occurrences of dyslipidaemia across various morphological patterns of lichen planus. Lopez-Jornet et al reported a higher prevalence of dyslipidaemia in oral LP patients. We could not find any significant association between various morphological patterns of oral lichen planus and dyslipidaemia. Lopez-Jornet et al found significantly higher 
triglyceride levels in patients with atrophic-erosive types than reticular types. In our study, 7 out of the 10 patients with nail involvement had dyslipidaemia (70.0\%). We could find a significant correlation between nail involvement and dyslipidaemia.

\section{Limitations of the Study}

The cross sectional nature of the study might not be helpful in establishing the true association between lichen planus and dyslipidaemia. Age of the study population ranged from 7-78 years where this might have acted as a confounding factor. The lipid profile status of the study population prior to the onset of lichen planus was not available.

\section{CONCLUSION}

41.3\% lichen planus patients presented with dyslipidaemia. Patients with extensive skin lesions had a higher prevalence of dyslipidaemia. There is no association of various morphological patterns of lichen planus with dyslipidaemia.

The exact prevalence of dyslipidaemia among lichen planus patients in India remains undocumented. Further studies are needed to establish the association between lichen planus and lipid abnormalities. The knowledge of lipid abnormalities in lichen planus patients will be useful in predicting cardiovascular risk factors in these patients and thereby helping in taking preventive measures.

\section{REFERENCES}

[1] Breathnach SM. Lichen planus and lichenoid disorders. In: Burns DA, Cox NO, Breathnach S, et al. eds. Rook's text book of dermatology. $8^{\text {th }}$ edn. Oxford: Blackwell 2010:41.1- 41.27.

[2] Arias-Santiago S, Buendía-Eisman A, AneirosFernández J, et al. Cardiovascular risk factors in patients with lichen planus. Am J Med 2011;124(6):543-8.

[3] Dreiher J, Shapiro J, Cohen AD. Lichen planus and dyslipidaemia: a case-control study. Br J Dermatol 2009;161(3):626-9.
[4] Arias-Santiago S, Buendía-Eisman A, AneirosFernández J, et al. Lipid levels in patients with lichen planus: a case-control study. J Eur Acad Dermatol Venereol 2011;25(12):1398-401.

[5] López-Jornet P, Camacho-Alonso F, RodríguezMartínes MA. Alterations in serum lipid profile patterns in oral lichen planus. A cross-sectional study. Am J Clin Dermatol 2012;13(6):399-404.

[6] Rasouli N, Kern PA. Adipocytokines and the metabolic complications of obesity. J Clin Endocrinol Metab 2008;93(11 Suppl 1):S64-73.

[7] Roberts CK, Sindhu KK. Oxidative stress and metabolic syndrome. Life Sci 2009;84(21-22):705-12.

[8] Grattagliano I, Palmieri VO, Portincasa P, et al. Oxidative stress-induced risk factors associated with the metabolic syndrome: a unifying hypothesis. J Nutr Biochem 2008;19(8):491-504.

[9] Aly DG, Shahin RS. Oxidative stress in lichen planus. Acta Dermatoven APA 2010;19(1):3-11.

[10] Briganti S, Picardo M. Antioxidant activity, lipid peroxidation and skin diseases. What's new? J Eur Acad Dermatol Venereol 2003;17(6):663-9.

[11] Sezer E, Ozugurlu F, Ozyurt H, et al. Lipid peroxidation and antioxidant status in lichen planus. Clin Exp Dermatol 2007;32(4):430-4.

[12] Meller S, Gilliet M, Homey B. Chemokines in the pathogenesis of lichenoid tissue reactions. J Invest Dermatol 2009;129(2):315-9.

[13] de Grossmann SMC, de Aguiar MC, Teixeira R, et al. Oral lichen planus and chronic hepatitis C: a controversial association. Am J Clin Pathol 2007;127(5):800-4.

[14] Simark-Mattssona C, Bergenholtza G, Jontell M, et al. Distribution of interleukin $-2,-4,10$, tumour necrosis factor- $\alpha$ and transforming growth factor- $\beta$ mRNAs in oral lichen planus. Arch Oral Biol 1999;44(6):499507. 